\title{
NOTAS SOBRE LA ZONA OCCIDENTAL DE LA PROVINCIA DE MADRID
}

\author{
$311: 330.191 .4: 301: 353$
}

por

Ignacio Ballester Ros

SUMARIO: I. INTRODUCCION.-II. EL TERRITORIO Y LA POBLACION.-III. EL SECTOR AGRARIO.-IV. LA INDUSTRIA. V. EL SECTOR DE SERVICIOS: 1. EL COMERCIO. 2. EL TRANSPORTE Y LAS COMUNICACIONES. 3. LA ENSEÑANZA. 4. LA SANIDAD. 5. LOS SERVICIOS PÚBLICOS MUNICIPALES. 6. EL TURISMO Y EL EQUIPAMIENTO DEL OCIO.

\section{INTRODUCCION}

La Cámara de Comercio e Industria de Madrid ha impulsado, a través de su Servicio de Estudios Económicos, una actividad muy encomiable, en orden al conocimiento de la realidad socioeconómica de la Provincia.

Ha publicado monografías anuales sobre La Economía española, de 1975 a 1980; sobre Economía regional, de 1970 a 1978-79, y sobre La Economía de Madrid, editada, de manera ininterrumpida, desde el año 1952. Además, ha realizado «estudios de localización», concretados en dos monografías: Localización, estructura y dinámica del Comercio de Madrid, publicada en 1973, y la Localización y estructura de la Industria de Madrid y su Area de Influencia, editada en el año 1975. 
Elabora también, cada dos meses, tres informes de coyuntura relativos, respectivamente, a los resultados de las encuestas realizadas sobre situación y perspectivas de la Industria y del Comercio de Madrid, así como del Comercio de la Zona Centro. Y, mensualmente, elabora y publica los Indicadores de Coyuntura de la Economía Española y la Coyuntura Económica Internacional.

Finalmente, ha realizado y publicado, de 1976 a 1980, seis estudios socioeconómicos referidos a Alcalá de Henares, Aranjuez, la Comarca Lozoya-Somosierra, a la estructura del Comercio y la Industria de Getafe, a la estructura del Comercio y de la Industria de la Zona Suroeste de Madrid y a la estructura y dotación del comercio minorista de Madrid y otras ciudades.

Cerrando el ciclo de estudios socioeconómicos de la Provincia de Madrid, acaba de publicar el Estudio socioeconómico de la Zona Occidental de Madrid.

Como se observa, la profusión de estudios, que cubren ya toda el área provincial, reviste un extraordinario interés para el conocimiento de la vida local, a nivel municipal, en la mayor parte de sus aspectos. Resaltemos la utilización de una muy rica y abundante información estadística, basada, en algunos casos, en encuestas especiales.

Centraremos esta crónica en el comentario de la última monografía reseñada, referente a la Zona Occidental de Madrid, que «constituye un conjunto formado por 47 Municipios, situados entre el centro y el oeste de la Provincia de Madrid, en su límite con las de Segovia y Avila, que, aun no teniendo características socioeconómicas similares, reúne una serie de factores que le dan cierta homogeneidad".

En dicha monografía se aborda el análisis de la infraestructuıa física de la Zona y de sus características demográficas y el estudio de los sectores económico, agrario e industrial, de construcción y vivienda, comercio y transportes, turismo y servicios económicos, públicos y comunitarios.

\section{EL TERRITORIO Y LA POBLACION}

La Provincia de Madrid, situada en el centro de la Península, tiene la forma de un triángulo equilátero y está cubierta al nordeste, norte y noroeste por una extensa zona montañosa, formada por las 
cordilleras de Somosierra y Guadarrama, con el pico de Peñalara como cima más elevada; y aun al oeste llegan las estribaciones de la Sierra de Gredos. El resto de la Provincia, aproximadamente la mitad de su superficie, es una meseta, de altura media algo superior a los 600 metros.

De ambas características participa la Zona Occidental de la Provincia, objeto de este trabajo de síntesis, surcada por los ríos Manzanares y Guadarrama, que discurren en dirección norte-sur, y por el Alberche, que inicialmente se orienta al este, para girar luego, ya dentro de la Provincia de Madrid, hacia el sudoeste.

Esta Zona tiene una extensión superficial de 2.223,6 kilómetros cuadrados, equivalente al 27 por 100 de la superficie provincial. Sobre ella se asienta una población de 129.774 habitantes, que supone el 3,12 por 100 de la población provincial o el 12 por 100 de la población del resto de la Provincia, sin el Municipio de Madrid. La densidad de población es de 58 habitantes por kilómetro cuadrado.

En el período 1900-1975 la población de la Zona se ha triplicado, mientras que la de la Provincia se ha quintuplicado. Ello supone una pérdida de la capacidad de fijación de la población, ya que a principios de siglo la población de la Zona era solamente de 46.090 personas, pero representaba el 5,94 por 100 de la población de la Provincia.

En el crecimiento demográfico de la Zona, en su conjunto, caben distinguir tres etapas: la primera, hasta 1930, de crecimiento lento, casi de estancamiento; la segunda, de crecimiento algo mayor, de 1930 a 1960; y la tercera, a partir de 1960, con un crecimiento neto muy acusado.

Las acusadas diferencias demográficas existentes en la Zona hace aconsejable subdividirla en Subzonas de cierta homogeneidad sucioeconómica y demográfica. Siguiendo a COPLACO, consideraremos las tres Subzonas siguientes:
A) Subzona Area Metropolitana.
B) Subzona Sierra Centro.
C) Subzona Sierra Sur.

La distribución de los 47 Municipios de la Zona en las expresadas Subzonas, con expresión de la población de cada uno, según el Padrón renovado de Habitantes de 1975, es ésta: 


\section{A) Subzona Area Metropolitana}

Boadilla del Monte $\ldots \ldots \ldots \ldots \ldots \ldots \ldots$

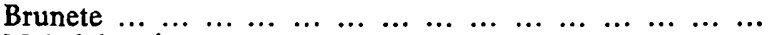

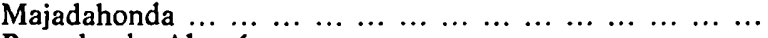

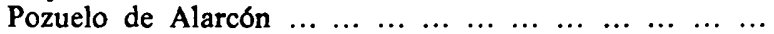

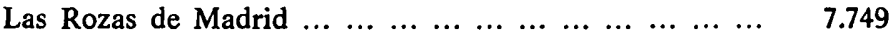

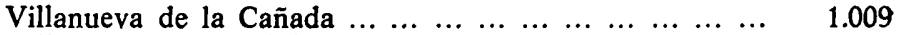

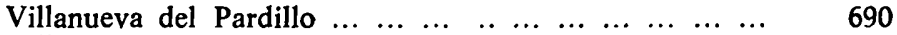

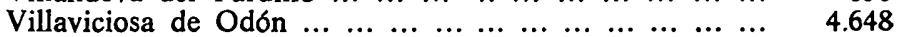

\section{B) Subzona Sierra Centro}

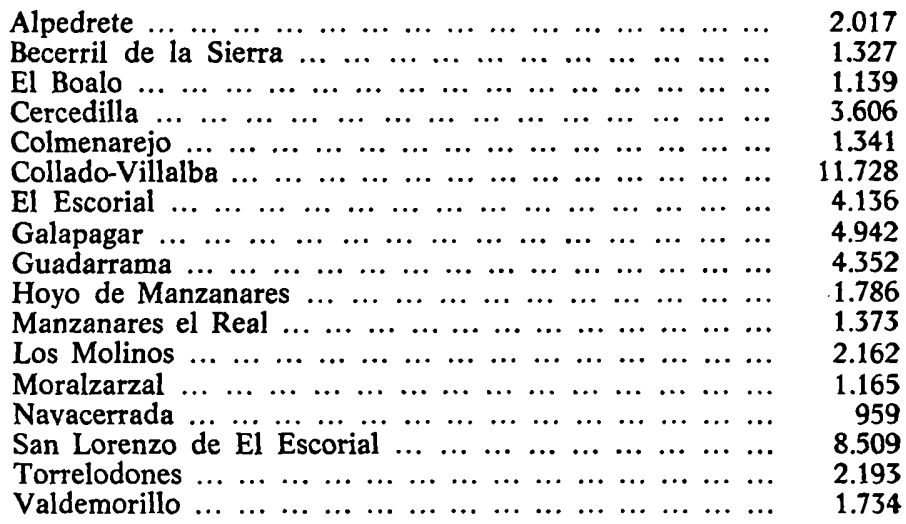

\section{C) Subzona Sierra Sur}

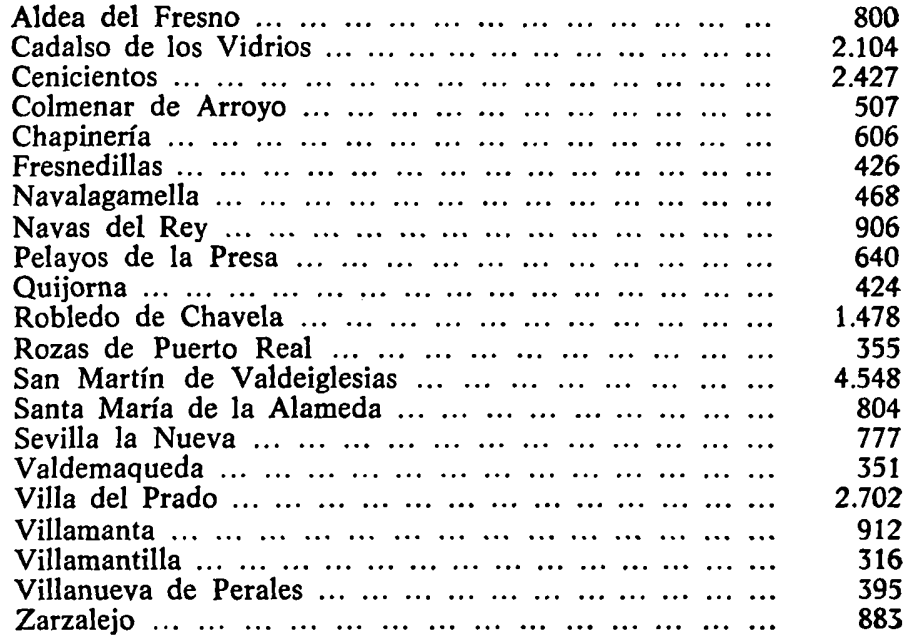


Podemos resumir la superficie y población de las tres Subzunas en esta forma:

\begin{tabular}{|c|c|c|c|c|}
\hline & Municipios & $\underset{\substack{\text { Superficie } \\
\mathrm{km}^{2}}}{ }$ & Poblacion & $\begin{array}{l}\text { Densidad } \\
\text { de } \\
\text { poblacion }\end{array}$ \\
\hline $\begin{array}{lllllll}\text { Subzona } & A & \ldots & \ldots & \ldots & \ldots & \ldots \\
\text { Subzona } & \text { B } & \ldots & \ldots & \ldots & \ldots & \ldots \\
\text { Subzona } & \mathbf{C} & \ldots & \ldots & \ldots & \ldots & \ldots \\
\end{array}$ & $\begin{array}{r}8 \\
18 \\
21\end{array}$ & $\begin{array}{r}363,3 \\
82405 \\
1.036,29\end{array}$ & $\begin{array}{l}51.405 \\
55.720 \\
22.649\end{array}$ & $\begin{array}{r}141,49 \\
67,62 \\
21,89\end{array}$ \\
\hline
\end{tabular}

Las Subzonas A y B tienen un volumen de población casi similar, con el 43 y el 40 por 100, respectivamente, de la población de la Zona. La Subzona C está peor poblada. El número de Municipios y la extensión superficial de cada Subzona están en razón inversa de la densidad de población de cada Subzona. La más densamente poblada es la Subzona A, que comprende ocho Municipios, todos ellos, salvo Las Rozas de Madrid, integrados en el Area Metropolitana. La Subzona B, que comprende el centro y norte de la Zona Occidental, que es la de expansión de Madrid y Area Metropolitana, en la forma generalizada de segunda residencia, tiene una densidad un poco inferior a la nacional. La densidad de la Subzona $C$, que comprende el suroeste de la Zona, es muy reducida.

La distribución de los Municipios de la Zona Occidental, en su conjunto, según su tamaño demográfico y su evolución en el periodo 1900-1975, ha sido el siguiente:

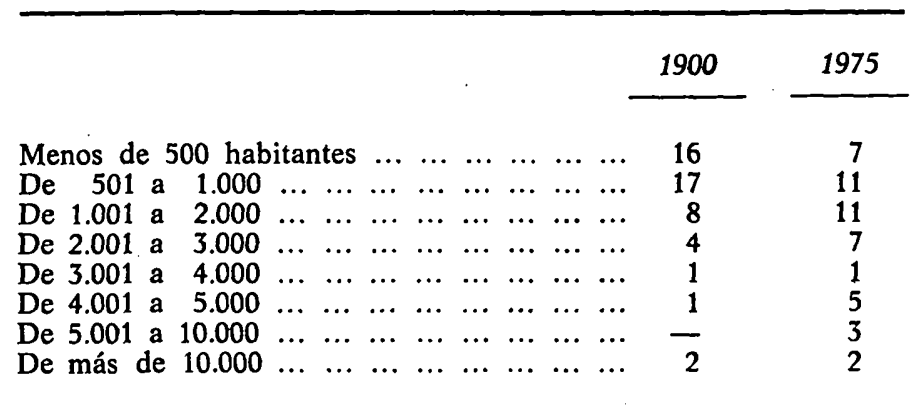

Se observa un proceso de concentración de la población hacia Municipios de mayor tamaño, de tipo urbano, con disminución del 
número de Municipios rurales, si bien en 1975 existen tıdavía 29 Municipios menores de 2.000 habitantes.

Se incrementa la importancia de los Municipios intermedios, de entre 2.001 y 10.000 habitantes, que pasan de 6 a 16 en dicho periodo, y surgen dos nuevos Municipios con población superior a los 10.000 habitantes.

Téngase en cuenta que en 1900 el 63 por 100 de la población residía en Municipios rurales y el 37 por 100 restante en Municipios intermedios, mientras que en 1975 el 20 por 100 de la población reside en Municipios rurales, el 53 por 100 en Municipios intermedios y el 27 por 100 en Municipios urbanos.

El crecimiento demográfico de la Provincia de Madrid cbedece primordialmente a una tendencia hacia la concentraciún de la población en Municipios urbanos que, en especial, configuran el Area Metropolitana, que ha determinado un desorbitado desarrollo de algunos Municipios; como consecuencia del flujo migratorio provincial e intraprovincial. Paralelamente se ha producido una pérdida de población en algunos Municipios, que han visto acentuada su ruralización. Estas características presenta también lá Zora Occidental de la Provincia.

La Subzona A. Area Metropolitana se ha quintuplicado desde el año 1930. Se ha incrementado la población de determinados Municipios urbanos, como Pozuelo de Alarcón, Majadahonda y Las Rozas de Madrid, que en 1975 tienen, en conjunto, 41.193 habitantes, la tercera parte de la población de la Zona Occidental.

Se da la circunstancia de que las personas activas residentes en estos tres Municipios ascendían a 25.514 en 1979, mientras que los puestos de trabajo existentes eran solamente 9.313. Había, pues, más de 16.000 personas que trabajaban en Municipios distintos al de su residencia, especialmente en Madrid.

La población activa de los Municipios de esta Subzona se caracteriza por un predominio de la juventud, con un promedio superior incluso al de la capital, debido a la fuerte inmigración, procedente de aquélla. Más de la mitad de activos pertenecen al sector de servicios y cerca de la quinta parte a la construcción. Una quinta parte de la población activa son profesionales o directivos, y hay un elevado porcentaje de personal obrero.

En la Subzona B. Sierra Centro la generalización de las segundas residencias y los desplazamientos de fin de semana desde la capital y su área han influido notoriamente en la transformación de 
su estructura económica y social. La población se ha duplicado, pasando de 24.096 en 1930 a 55.720 en 1975, si bien el peso de la Subzona en el conjunto de la Zona Occidental se mantiene inalterable.

El análisis de la pirámide de población permite afirmar que existe un doble movimiento migratorio. De una parte, una corriente emigratoria de la Subzona hacia Madrid y su Area Metropolitana, que afecta especialmente a las mujeres; y de otra, un proceso inmigratorio, que procede de Provincias limítrofes a Madrid.

Existe una estrecha relación entre las características de la población activa de la Subzona y las necesidades de expansión y ocio del Area Metropiltana de Madrid y de la propia capital. Predomina el sector de la construcción, seguido de los servicios comunales, sociales y personales, y en segundo término, la mano de obra del sector comercio e industrias manufactureras. Desde el punto de vista de la profesión, casi las dos terceras partes pertenecen a oficios varios, especialmente industriales y del transporte y peones no agrarios, seguidos, a lo lejos, del personal de servicios y de comerciantes y agricultores.

La población activa femenina asciende al 16 por 100 , ocupando el primer lugar los servicios, las profesionales liberales $y$, en tercer término, el personal administrativo y empleado en el comercio.

En la Subzona $C$. Sierra Sur la población ha descendido en el período 1930-1975. La mitad de los Municipios sufren una recesión demográfica, mostrando una situación de despoblación, debido a la corriente emigratoria hacia otros Municipios de la Provincia, especialmente a la capital. Se reduce el volumen de la población activa y crece el número de los de edad avanzada, denotando un progresivo envejecimiento de la población. Por otra parte, se advierte desequilibrio en la distribución por sexos, al igual que en la Subzona B, en los grupos de jóvenes, adultos y viejos, aunque en proporción distinta.

En la población activa ocupan los primeros lugares los agricultores, el personal dedicado a la construcción y los de servicios comunales, sociales y personales. En cuanto a la profesión predominan los agricultores y ganaderos y el personal de oficio.

La participación de la mujer en la población activa se cifra en el 12 por 100 , y trabajan en los servicios comunales, sociales y personales, ejercen profesiones liberales o son personal administrativo. 


\section{EL SECTOR AGRARIO}

La actividad agraria no tiene demasiada importancia en esta Zona. La agricultura es predominantemente de secano, con baja productividad, deficiente parcelación y capitalización y escasa organización comercial. La superficie cultivada en regadío es muy pequeña y no son de esperar grandes transformaciones. La ganadería está poco desarrollada, aunque destaca el ganado bovino en la Subzona Sierra Centro y el ovino y el caprino en la Subzona Sierra Sur, y es susceptible de una notable reconversión que ponga en marcha grandes explotaciones, basadas en la abundancia de pastos y en el aumento de la producción de forrajes. La producción de madera es reducida y no se vislumbran grandes cambios.

La superficie de la Zona se distribuye de la siguiente forma:

\begin{tabular}{|c|c|c|}
\hline & Has. & $\%$ \\
\hline 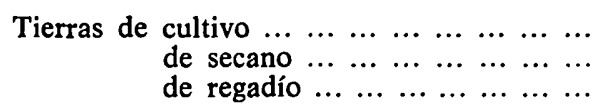 & $\begin{array}{r}38.262 \\
37.099 \\
1.163\end{array}$ & 17,2 \\
\hline 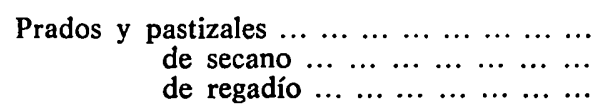 & $\begin{array}{r}46.565 \\
45.258 \\
1.307\end{array}$ & 20,9 \\
\hline Terreno forestal $\ldots \ldots \ldots \ldots \ldots \ldots \ldots \ldots$ & 74.840 & 33,7 \\
\hline 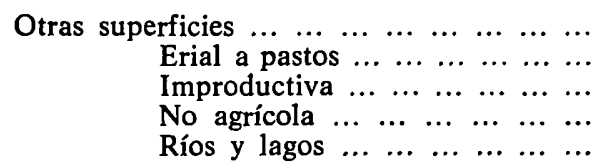 & $\begin{array}{r}62.693 \\
31.139 \\
7.544 \\
20.500 \\
3.510\end{array}$ & 28,2 \\
\hline
\end{tabular}

En relación con la Provincia en su conjunto, la Zona resulta pobre en tierras de cultivo, tanto en secano como en regadío; pero es proporcionalmente su riqueza en prados y pastizales y también en terreno forestal, ya que la mitad de la superficie forestal de la Provincia corresponde a esta Zona.

Según el Censo Agrario de 1972, elaborado por el Instituto Nacional de Estadística, había en la Zona 7.353 explotaciones agrarias, el 29,3 por 100 del total de la Provincia, 322 de ellas puramente ganaderas. El 60 por 100 de las explotaciones tienen menos de 5 Has., el 35 por 100 oscilan entre 5 y 100 Has. y el 5 por 100 son mayores: 
de 100 Has. Las grandes explotaciones predominan en la Subzona Sierra Centro y las pequeñas en la Subzona Sierra Sur.

La población activa agraria de la Zona representa menos del 10 por 100 de la total y ofrece un alto grado de envejecimiento. Existen, sin embargo, algunos Municipios de la Subzona Sierra Sur en que la población activa agraria supera el 50 por 100 y otros de la Subzona Sierra Centro con entre el 20 y el 35 por 100 de la población activa total. En los Municipios de la Subzona Area Metropolitana la población activa agraria, con alguna excepción, tiene menor importancia relativa.

En la mayor parte de los Municipios de la Subzona Sierra Centro las tierras de cultivo suponen el 5 por 100 de la superficie total. Lo contrario ocurre en la Subzona Sierra Sur, con más del 40 por 100 de tierras de cultivo. Los Municipios de la Subzona Area Metropolitana tienen, salvo excepciones, poca superficie de cultivo.

Las tierras de cultivo se distribuyen así:

\begin{tabular}{|c|c|c|c|}
\hline & & Has. & $\%$ \\
\hline Cultivos & 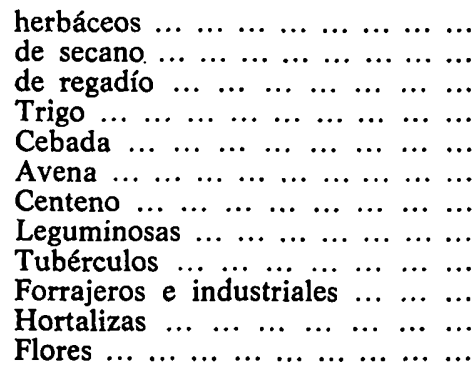 & $\begin{array}{r}9.294 \\
8.367 \\
927 \\
4.952 \\
1.777 \\
754 \\
218 \\
431 \\
127 \\
495 \\
447 \\
10\end{array}$ & 24,3 \\
\hline \multicolumn{2}{|c|}{ Barbechos ... } & 16.192 & 42,3 \\
\hline Cultivos & 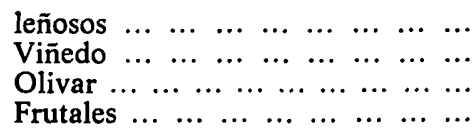 & $\begin{array}{r}12.771 \\
11.213 \\
1.271 \\
287\end{array}$ & 33,4 \\
\hline
\end{tabular}

Los cultivos herbáceos de la Zona tienen menor importancia relativa que en el conjunto de la Provincia; sin embargo, la Zona sobresale por los cultivos leñosos. Los barbechos están a nivel casi análogo al de la Provincia.

Entre los cultivos herbáceos destacan los cereales, junto a los demás grupos de cultivos, cuya superficie cultivada se ha consigna- 
do. La zona cerealista más importante está formada por los Municipios de Villaviciosa de Odón y Brunete —en la Subzona Area Metropolitana- y sus colindantes de Quijorna, Villanueva de Perales. y Villamanta. Sólo en centeno destaca Manzanares el Real. Los tubérculos y las hortalizas se cultivan primordialmente en Villaviciosa de Odón y Villa del Prado. Las leguminosas —algarroba, esencialmente-, en Villamanta. Los cultivos forrajeros - alfalfa y vezacorresponden a Brunete, El Escorial y Aldea del Fresno. Y las flores se cultivan en Villaviciosa de Odón.

En cuanto a los cultivos leñosos, ocupa el primer lugar el viñedo, destinado a la producción de uva para vinificación, que se sitúa en los Municipios de San Martín de Valdeiglesias, Cadalso de los Vidrios, Cenicientos y Villa del Prado, prolongándose, con menor intensidad, por Navas del Rey, Aldea del Freno y Villamanta, que, sin solución de continuidad, constituyen la zona vitivinícola en la Subzona Sierra Sur. Esta misma zona es la del olivar, destinado a la. producción de aceite.

Los frutales, aparte de las plantaciones regulares, se cultivan mucho en diseminado. Los principales son el almendro y el manzano, que se cultivan principalmente en los Municipios de San Martín de Valdeiglesias y Villamanta. Igualmente hay cultivos de peral, cerezo e higueras.

La cabaña ganadera de la Zona está constituida por el siguiente número de cabezas:

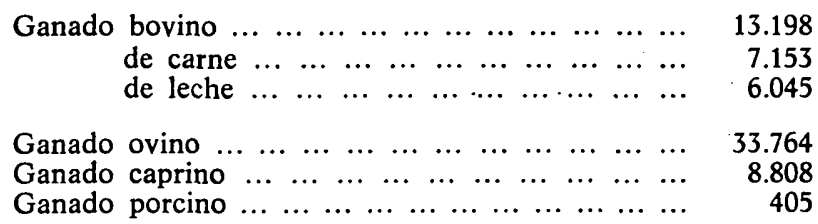

El ganado bovino es el que reviste mayor importancia, por la. abundancia de pastos, si bien las explotaciones son pequeñas. Los Municipios de Cercedilla, Guadarrama y Valdemorillo, de la Subzona Sierra Centro, y de Santa María de la Alameda y Villanueva de Perales, de la Subzona Sierra Sur, sobresalen en la ganadería bovina de carne; y Cercedilla y Valdemorillo, en la de leche.

Se registra una regresión en los ganados ovino y caprino, debido. a la mano de obra que requiere y a lo poco rentable de las explota- 
ciones. El ganado ovino destaca en los Municipios de Collado-Villalba, Hoyo de Manzanares, Moralzarzal y Valdemorillo, en la Subzona Sierra Centro; y en los de Santa María de la Alameda, Villanueva de Perales, Villamanta y Villa del Prado.

El ganado caprino se halla principalmente en los Municipios de Becerril de la Sierra y Santa María de la Alameda; y el porcino, en Valdemorillo y Villa del Prado. Existe ganado caballar en Santa María de la Alameda.

Hay algunas granjas avicolas en los Municipios de Alpedrete, Colmenarejo, Valdemorillo y Fresnedillas.

En cuanto a la riqueza forestal, predomina el monte abierto, siguiéndole en importancia el maderable y el leñoso, estos dos últimos en proporción casi similar. La especie predominante es el pino.

La riqueza forestal de la Zona se extiende a las tres Subzonas. En la Subzona Sierra Centro comprende los Municipios de Guadarrama, Los Molinos, Cercedilla, Navacerrada, Manzanares el Real, Moralzarzal, Collado-Villalba, Hoyo de Manzanares y Galapagar. En la Subzona Area Metropolitana abarca los Municipios de Boadilla del Monte, Pozuelo de Alarcón y Las Rozas de Madrid. Y en la Subzona Sierra Sur comprende los Municipios de Valdemaqueda, Robledo de Chavela, Fresnedillas, Navalagamella, Colmenar del Arroyo, Navas del Rey, Villamantilla, Aldea del Fresno, Villa del Prado; San Martín de Valdeiglesias y Cenicientos.

\section{LA INDUSTRIA}

La actividad industrial tiene relativamente poca importancia en la Zona. La Casa de Campo y las zonas colindantes se interponen en el desarrollo industrial, bajo la influencia de Madrid, hacia el norte. y el oeste. De otra parte, las zonas residenciales, que se iniciaron muy tempranamente, motivadas por las extraordinarias condiciones naturales, paisajísticas y climatológicas de la Zona, determinaron una gran demanda de terrenos de suelo calificado, de altos precios, que desalentaron su adquisición a fines industriales.

Excepción hecha del sector de la construcción, la actividad propiamente dicha de producción es reducida, predominando las de instalación, reparación y almacenaje. En 1979 se registra la existencia de 3.061 contribuyentes industriales, el 5,5 por 100 del total pro- 
vincial. Con más de 100 contribuyentes había nueve Municipios: Pozuelo de Alarcón, Las Rozas de Madrid y Majadahonda, en la Subzona Area Metropolitana, y Collado-Villalba, Galapagar, San Lorenzo de El Escorial, Cercedilla, El Escorial y Valdemorillo, en la Subzona Sierra Centro. Los Municipios de más rápido desarrollo industrial han sido Valdemorillo y Majadahonda.

Atendiendo sólo a la actividad principal, según los epígrafes de la Licencia Fiscal, la distribución por ramas y Subzonas es ésta:

\begin{tabular}{|c|c|c|c|c|}
\hline & Total & $\begin{array}{c}\text { Area Me- } \\
\text { tropolitana }\end{array}$ & $\begin{array}{l}\text { Sierra } \\
\text { Centro }\end{array}$ & $\begin{array}{c}\text { Sierra } \\
\text { Sur }\end{array}$ \\
\hline En conjunto $\ldots \ldots \ldots$ & 755 & 262 & 363 & 140 \\
\hline 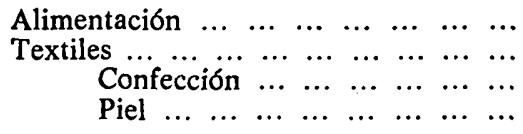 & $\begin{array}{r}118 \\
52 \\
18 \\
34\end{array}$ & $\begin{array}{r}26 \\
31 \\
9 \\
22\end{array}$ & $\begin{array}{r}51 \\
17 \\
7 \\
10\end{array}$ & $\begin{array}{r}41 \\
4 \\
2 \\
2\end{array}$ \\
\hline 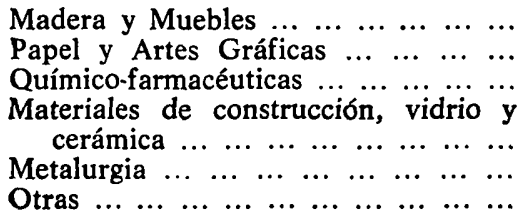 & $\begin{array}{r}116 \\
18 \\
28\end{array}$ & $\begin{array}{r}15 \\
114 \\
5\end{array}$ & $\begin{array}{r}54 \\
8 \\
10\end{array}$ & $\begin{array}{l}12 \\
64 \\
-\end{array}$ \\
\hline
\end{tabular}

En la Subzona Area Metropolitana, cinco Municipios absorben casi el 90 por 100 de los establecimientos industriales: Pozuelo de Alarcón (90), Majadahonda (49), Las Rozas de Madrid (39), Villaviciosa de Odón y Boadilla del Monte (27 cada uno).

En Pozuelo destacan las industrias de maquinaria, matadero frigorífico, papel y artes gráficas, plásticos, piel y confección y muebles. En Majadahonda, plásticos, componentes de electrodomésticos, hormigón y quesos. En Las Rozas, muebles y panificadora. En Boadilla, materiales de construcción y artes gráficas. En Villaviciosa, materiales de construcción. $Y$ en todos ellos, numerosos talleres de reparación de automóviles, que cubren prácticamente la rama de metalurgia.

En la Subzona Sierra Centro, más importante por el número de establecimientos, también una gran parte de los mismos -el 60 por 100 - se hallan concentrados en cinco Municipios: Collado-Vi- 
llalba (77), San Lorenzo de El Escorial (44), Guadarrama (27), Alpedrete (26) y El Escorial (25).

En Collado-Villalba sobresalen las industrias de materiales de construcción, productos químicos, casas prefabricadas de madera y panificadora. En San Lorenzo de El Escorial, muebles y carpintería mecánica. En Guadarrama, madera, alimentación y bebidas. En todos ellos, talleres de reparación de automóviles. En Collado Mediano hay fábrica de fertilizantes.

En la Subzona Sierra Sur, con menor número de establecimientos, destacan San Martín de Valdeiglesias, Sevilla la Nueva y Navas del Rey, con industrias de cerrajería, metalurgia, muebles y materiales de construcción.

En cuanto a la industria de la construcción, esta Zona constituye un tradicional mercado de construcción de viviendas, con predominio de la iniciativa privada y abundancia de pequeñas promociones, si bien en los últimos años se advierte una contracción de la demanda.

En 1980 había en la Zona 296 constructores, en general pequeñas y medianas empresas, que atendían a las necesidades de viviendas.

En el mismo años existían en la Zona:

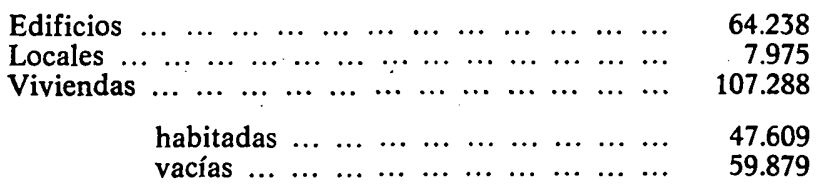

El número de viviendas vacías es grande, debido a la ubicación en la Zona de la mayor parte de las viviendas de segunda residencia, fenómeno que ha permitido un gran desarrollo económico de los Municipios, contribuyendo al crecimiento de la población y del nivel de vida y también al incremento de las actividades comerciales y, en general, del sector de servicios.

En 1979 se habían construido en la Provincia de Madrid 66.000 unidades de segunda residencia y se hallaban en construcción 7.000. El chalet individual es el tipo más generalizado, con el 52 por 100 del total, seguido de los apartamentos, el 38 por 100, y de los chalets adosados. 
En la Zona existían las siguientes viviendas de segunda residencia:

\begin{tabular}{|c|c|c|c|c|}
\hline & & Apartamentos & $\begin{array}{c}\text { Chalets } \\
\text { individuales }\end{array}$ & $\begin{array}{l}\text { Chalets } \\
\text { adosados }\end{array}$ \\
\hline & 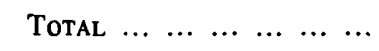 & 20.122 & 20.447 & 4.126 \\
\hline Subzona & Area Metropolitana ... ... & - & 3.726 & 1.704 \\
\hline Subzona & Sierra Centro $\ldots \ldots \ldots$ & 19.300 & 10.973 & 2.275 \\
\hline Subzona & Sierra Sur $\ldots \ldots \ldots \ldots$ & 822 & 5.748 & 147 \\
\hline
\end{tabular}

En la Subzona Area Metropolitana destacan, por el número de viviendas de segunda residencia, los Municipios de Pozuelo de Alarcón, Las Rozas de Madrid y Majadahonda.

En la Subzona Sierra Centro, la de mayor importancia, sobresalen Collado-Villalba, con 9.000 viviendas; Guadarrama, con 5.000; Alpedrete, Hoyo de Manzanares y Galapagar, con más de 2.000 cada uno, y Becerril de la Sierra, Moralzarzal, Cercedilla, Manzanares el Real, El Boalo, El Escorial y Valdemorillo, con más de 1.000 cada uno.

En la Subzona Sierra Sur, la menos importante en este orden, sobresalen Pelayos de la Presa, y se acercan a este límite Robledo de Chavela, Villa del Prado y San Martín de Valdeiglesias.

\section{EL SECTOR DE SERVICIOS}

1. El COMERCIO

La estructura del comercio está íntimamente ligada a la estructura demográfica y urbanística. En la Zona existían en 1980450 licencias comerciales de mayoristas y 4.292 de minoristas, que, por sectores, se distribuyen de esta forma: 


\begin{tabular}{|c|c|c|}
\hline & Mayoristas & Minoristas \\
\hline 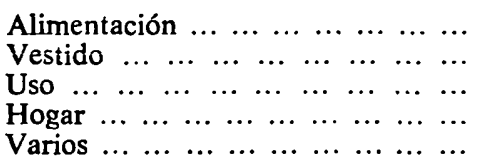 & $\begin{array}{r}75 \\
4 \\
28 \\
14 \\
329\end{array}$ & $\begin{array}{r}1.560 \\
201 \\
481 \\
316 \\
1.734\end{array}$ \\
\hline
\end{tabular}

En varios se incluyen restaurantes, cafeterías, bares y tabernas.

El equipamiento comercial predominante es todavía el tradicional, consistente en establecimientos de pequeñas dimensiones, de tipo familiar, con reducido volumen de venta y escasez de recursos disponibles.

En los últimos años han entrado en funcionamiento establecimientos comerciales en régimen de libre servicio, la mitad inaugurados en el período 1961-1972 y la otra mitad a partir de 1972. De ellos, hay 77 autoservicios, 24 superservicios, con 10.000 metros cuadrados de superficie de venta, y dos hipermercados, estos últimos en Majadahonda y Las Rozas de Madrid. Los autoservicios se hallan situados en El Escorial, Guadarrama, Collado-Villalba, Manzanares el Real y Becerril de la Sierra, y los superservicios, en Majadahonda, Guadarrama, Collado-Villalba, Pozuelo de Alarcón, San Lorenzo de El Escorial, Las Rozas de Madrid y Valdemorillo, es decir, en las Subzonas Area Metropolitana y Sierra Centro.

\section{EL TRANSPORTE Y LAS COMUNICACIONES}

El territorio de la Zona está atravesado por cinco carreteras principales: la de Madrid a La Coruña, que registra altas densidades de tráfico, con 87.439 vehículos diarios de media en Aravaca, 55.354 en Las Rozas, 36.500 en Las Matas y 5.827 en el Alto de los Leones; la desgajada de la anterior, de Villalba a Segovia, con una intensidad de 3.366 vehículos en el Puerto de Navacerrada; la de Las Rozas a El Escorial, con 8.430 vehículos diarios; la de Navalcarnero a Navacerrada, por San Lorenzo de El Escorial, y la de Madrid a San Martín de Valdeiglesias, todas ellas en perfecto estado. Las carreteras secundarias están en buen estado en la Subzona Sierra Centro, en regular estado en la Subzona Area Metropolitana y en estado deficiente en la Subzona Sierra Sur. 
La red de líneas de autobuses que unen a Madrid con los Municipios de la Zona comprende 21 líneas principales, con un recorrido de 1.531 kilómetros y una frecuencia diaria, en días laborables, de entre dos y siete viajes, necesidades e intensidades que se incrementan de manera notoria en la época estival, aunque se advierte insuficiencia de servicio en algunos Municipios. El servicio puede calificarse de bueno en toda la Subzona Sierra Centro y en algunos Municipios de las Subzonas Area Metropolitana y Sierra Sur.

$\mathrm{El}$ servicio ferroviario está físicamente limitado a aquellos $\mathrm{Mu}$ nicipios que disponen de estación ferroviaria o están próximos y tienen buenas comunicaciones de autobuses por carretera con las estaciones ferroviarias. El servicio se estima como muy bueno en la Subzona Area Metropolitana, regular en la Subzona Sierra Centro e insuficiente en la Subzona Sierra Sur.

Los servicios telefónico, postal y telegráfico se califican de buenos en la Subzona Sierra Centro e insuficientes en las otras dos Subzonas.

\section{LA ENSEÑANZA}

En el curso 1978-79 han funcionado 132 centros de enseñanza, 85 estatales y 47 no estatales, con un total de 1.247 unidades escolares, de ellas 579 correspondientes a centros estatales y 668 a centros no estatales. En conjunto, suponen 49.750 puestos escolares, 23.740 estatales y 26.010 no estatales.

La distribución de los centros estatales es similar en las tres Subzonas; no así la de unidades y puestos escolares, que descienden claramente en la Subzona Sierra Sur, habida cuenta de la menor densidad demográfica de sus Municipios.

En cuanto a los centros no estatales, destacan en la Subzona Area Metropolitana, donde se ubican más de la mitad de ellos (28), con 564 unidades y 21.885 puestos escolares, lo que equivale casi a la mitad de los puestos escolares de la Zona.

Es Pozuelo de Alarcón el Municipio en que se encuentra la mayor parte de los centros no estatales (16), con 300 unidades. Tanto en este Municipio como en los de Las Rozas de Madrid y Boadilla del Monte se atiende en sus centros tanto a la población escolar propia como a la de Madrid. 
Los alumnos matriculados son: 12.794 en la Subzona Sierra Centro, 6.997 en la Subzona Sierra Sur y 25.612 en la Subzona Area Metropolitana. Representan una cifra inferior a la de puestos escolares, salvo en la Subzona Sierra Sur, en la que el número de alumnos por clase es superior al normal.

Por niveles de estudio, en el conjunto de la Zona, el 16,1 por 100 del alumnado corresponde a Educación Preescolar; el 83,7, a Educación General Básica, y el 0,2 por 100, a Educación Especial y de Adultos.

\section{LA SANIDAD}

Se registra una distribución irregular de los servicios sanitarios, concentrándose en Madrid la mayor parte de ellos, mientras que el resto de la Provincia presenta insuficiencias por hallarse por debajo de la media nacional en la disponibilidad de estos servicios.

En la Zona Occidental el nivel sanitario es aceptable, aunque en algunos núcleos no sean los adecuados, debido a la dispersión de la población. Se dispone de 78 médicos y 48 farmacias, aunque hay 10 Municipios con médico compartido y 15 sin farmacia.

Existen dispensarios de la Seguridad Social en San Lorenzo de El Escorial, Collado-Villalba, Las Rozas de Madrid y Pozuelo de Alarcón, 28 clínicas rurales y siete Casas del Médico.

Destaca entre todos Pozuelo de Alarcón, con 22 médicos y ocho farmacias.

\section{LOS SERVICIOS PÚBLICOS MUNICIPALES}

En orden al abastecimiento de agua, la Zona está atendida, en parte, por el Canal de Isabel II, que abastece al Area Metropolitana de Madrid, y por los sistemas de la Cuenca Alta y de la Cuenca Baja del Guadarrama.

En la Zona se hallan ubicados los embalses de Navacerrada, Santillana, La Jarosa, Navalmedio, Valmayor, Las Picadas y San Juan.

La situación resulta comprometida por la escasez de agua embalsada y el consumo diario, que se cifra en 102.240 metros cúbicos, 
muy especialmente por la afluencia masiva de visitantes en fines de semana y en la época estival.

Se califica de bueno el servicio de energa eléctrica en las Subzonas Sierra Centro y Sierra Sur, y simplemente bueno, con insuficiencias, en la Subzona Area Metropolitana.

Son bastantes las insuficiencias en el alumbrado público, si bien en fase de ejecución se hallan bastantes proyectos de ampliación y mejora en las tres Subzonas.

El alcantarillado se estima regular en la Subzona Area Metropolitana y bueno, con algunas deficiencias, en las otras dos Subzonas.

Existen muchos proyectos de mejora del pavimentado, pero, salvo en la Subzona Area Metropolitana, en el resto es regular e insuficiente en el 60 por 100 de los Municipios.

\section{El TURISMO Y EL EQUIPAMIENTO DEL OCIO}

Desde hace muchos años, la Subzona Sierra Centro ha mantenido una gran tradición veraniega, cuyo principal foco fue San Lorenzo de El Escorial. Más tarde se ensancha la zona veraniega a casi todos los Municipios de la Zona, aunque no con un desarrollo homogéneo. $\mathrm{Y}$ surgen las construcciones urbanísticas, a las que ya nos hemos referido, en forma de chalet unifamiliar, chalets adosados y apartamentos, en la última fase. Sin embargo, no ha habido uniformidad en el desarrollo de las tres Subzonas, ni ha sido el turismo el único motivo del avance urbanístico, aunque el desarrollo de los servicios, que hemos descrito, ha estado motivado por el turismo o por las necesidades residenciales.

En la Subzona Area Metropolitana, la evolución turística se encaminaba a la segunda residencia, pero también a residencia habitual fuera de la capital, llegándose a construir edificios de viviendas compartidas para ambos fines. Debe tenerse en cuenta que la Subzona registra un notable incremento de población, que proviene de residentes en la capital con ciertos niveles de renta.

En la Subzona Sierra Sur, salvo en los parajes del Pantano de San Juan, el desarrollo de la segunda vivienda es más reciente y de menor entidad, aunque se prevé un notable auge en el futuro inmediato.

En la Subzona Sierra Centro la infraestructura turística está determinada por varios factores. En primer lugar, el paisaje, con la 
Sierra de Guadarrama, con parajes privilegiados como el Valle de la Fuenfría, Siete Picos, La Maliciosa y La Pedriza, y, en segunda línea, Hoyo de Manzanares, San Martín de Valdeiglesias, Abantos y Cenicientos.

En segundo término, los recursos de esparcimiento. En este orden los parajes de mayor calidad son El Escorial, Santa Maria de la Alameda, Cadalso de los Vidrios y el Pantano de San Juan.

En tercer lugar, existen lugares de preferente interés como conjuntos históricos, artísticos, núcleos de interés en que se integran elementos singulares, estaciones de esquí y otros centros deportivos.

Téngase presente que la Zona atrae cada verano, y en los fines de semana, a casi 600.000 personas, que constituyen la población flotante de la Zona, cuyos principales núcleos son Collado-Villalba, con 75.000; Cercedilla y Pozuelo de Alarcón, con 45.000; Guadarrama, con 40.000; Galapagar, con 35.000; Alpedrete y Manzanares el Real, con 25.000; Becerril de la Sierra, con 20.000, juntamente con Hoyo de Manzanares y Moralzarzal, y en cantidades inferiores los demás Municipios deda Zona.

La oferta turística de la Zona se concreta en 91 establecimientos hoteleros y 263 restaurantes. Más de la mitad de ellos se encuentran en la Subzona Sierra Centro. La Subzona Area Metropolitana tiene bastantes restaurantes; pero dispone de menos establecimientos hoteleros. Los principales establecimientos se concentran en Cercedilla, Navacerrada, Guadarrama, Collado-Villalba, Pozuelo de Alarcón, Majadahonda, San Lorenzo de El Escorial y San Martín de Valdeiglesias.

Campings y zonas de acampada son abundantes en Manzanares el Real, Cercedilla, El Escorial, Valdemorillo, Navalagamella, Navas del Rey, Aldea del Fresno, Pelayos de la Presa y Cadalso de los Vidrios.

Centros socioculturales, como bibliotecas y centros culturales, predominan en las Subzonas Area Metropolitana, Sierra Centro y Sierra Sur, ya que no existen sólo en dos Municipios de la primera y en seis de la segunda y en cinco de la última. En conjunto se dispone de 23 bibliotecas y 14 centros culturales.

El equipamiento deportivo presenta características similares en las tres Subzonas, aunque con una menor dotación en la Subzona Sierra Sur y una preferencia de centros equipados para los deportes de nieve en la Subzona Sierra Centro. Campos de deportes existen en todos los Municipios, con la excepción de nueve. Hay 11 cam- 
pings, 29 piscinas, 21 cines, 43 salas de baile, 19 billares y 11 bingos en el conjunto de la Zona, con preferente localización en la Subzona Sierra Centro.

La información numérica que contiene la monografía que hemos comentado es copiosísima y muy bien seleccionada. En ella pueden los estudiosos ampliar el conocimiento de las características que hemos contemplado.

Son de elogiar los estudios de esta naturaleza que se dedican al análisis de áreas geográficas que, por diversas motivaciones, ofrecen interés para su conocimiento en el área local, y es de desear que proliferen esta clase de trabajos. 


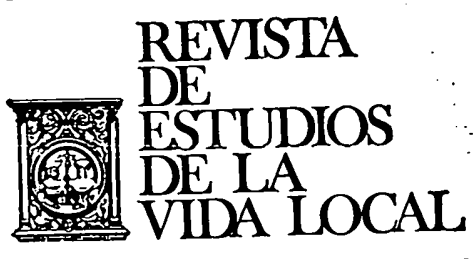

IV. JURISPRUDENCIA 
REVL-1981, núm. 212. BALLESTER ROS, IGNACIO. NOTAS SOBRE LA ZONA OCCIDENTAL DE L... REVL-1981, núm. 212. BALLESTER ROS, IGNACIO. NOTAS SOBRE LA ZONA OCCIDENTAL DE L... 